\title{
Basic human values: Inter-value structure in memory is
}

\author{
Ali Pakizeh, Jochen E. Gebauer, Gregory R. Maio* \\ Cardiff University, School of Psychology, Park Place, Cardiff, Wales CF10 3AT, UK
}

Received 28 April 2005; revised 20 December 2005

Available online 16 June 2006

\begin{abstract}
Three experiments examined the latent structure of values. Participants rated the importance of values clustered in pairs. Based on [Schwartz, S. H. (1992). Universals in the content and structure of values: Theoretical advances and empirical tests in 20 countries. In M. Zanna (Ed.), Advances in Experimental Social Psychology (Vol. 25, pp. 1-65). San Diego, CA: Academic Press.] circular model, we predicted and found that the time to rate the second value in each pair was shorter when the two values were motivationally congruent or opposing than when the two values were unrelated (Experiment 1). As expected, this was not the case when participants had to compare the importance of values within each pair (Experiment 2). Finally, semantic relatedness between values failed to explain the effects of motivational compatibility (Experiment 3). Taken together, these results reveal a coherent pattern of value relations driven by motivational compatibilities, over and above perceived semantic relatedness.
\end{abstract}

(C) 2006 Elsevier Inc. All rights reserved.

Keywords: Values; Attitudes; Memory; Inter-attitude structure

\section{Introduction}

The topic of the present research is at the core of many debates over social issues and policy. In these debates, people often disagree about the compatibility of different social and policy aims. Can you promote achievement by being more generous to the needy (e.g., by increasing social benefits)? Can you promote personal freedoms at the same time as attempting to protect national security (e.g., through anti-terror legislation)? Can you promote freedom of choice while protecting the sanctity of life (e.g., in issues of euthanasia, abortion, and animal rights). The answers to these questions depend on access to an underlying mental representation of how different values and ideals are interrelated. Our view is that, for the most part, people possess a common representation of these compatibilities and con-

\footnotetext{
We thank Lee Fabrigar, Geoff Haddock, Tony Manstead, Russell Spears, Ulrich von Hecker, and three anonymous reviewers for their comments at different stages of the research.

* Corresponding author.

E-mail address: maio@cardiff.ac.uk (G.R. Maio).
}

flicts, and the frequent debates arise primarily because of differences in the relative importance of values and the methods of pursuing them. The present research identifies important limitations of past efforts to uncover such a latent mental representation of values and attempts to identify this representation more powerfully.

\section{Values and Inter-Value Structure}

All aspects of individuals' everyday lives are influenced by human values (e.g., freedom and equality). People rely on these values by using them implicitly or explicitly to determine their future directions and justify their past actions, compare themselves with others, praise or blame themselves and others, take certain actions over other people and influence them, and rationalize their attitudes and behaviour (Feather, 1992; Rohan, 2000; Rokeach, 1973; Schwartz, 1992). Indeed, this view is built into two of the most widely used definitions of values: Rokeach (1973, p. 5) proposed that "values are enduring beliefs that a specific mode of conduct or end-state of existence is personally or socially preferable to an opposite or converse mode of 
conduct or end-state of existence", and Schwartz (1994, p. 21) indicated that values are "desirable transsituational goals, varying in importance, that serve as guiding principles in the life of a person or other social entity". Building on these views, research has found that values are important bridging constructs between personality traits and attitudes (Olson \& Maio, 2003; Yik \& Tang, 1996), and are among the most important predictors of behaviour and attitudes (Bardi \& Schwartz, 2003; Maio \& Olson, 1995; Rokeach, 1973). Research has also suggested that values are somewhat stable and resistant to change (Rokeach, 1985; Rokeach \& Ball-Rokeach, 1989) but nonetheless sensitive to contextual influences (e.g., Maio \& Olson, 1998). Furthermore, values are inter-generationally transmitted (Schonpflug, 2001; Whitbeck \& Gecas, 1988), and cherished across cultures (Bardi \& Schwartz, 2003; Schwartz, 1994).

Although the enormous set of findings related to values leaves no doubt about the influence of basic values on almost all aspects of human life, it may seem difficult to interpret the findings within a single nomological net. Interpretation requires a theory about the structure (representation) of values and value conflicts in the human mind. Early models about the structure of values (e.g., Feather, 1975; Rokeach, 1973) laid the groundwork for a comprehensive structural model provided by Schwartz (1992). The starting point for this model is the assumption that values are representations of three universal requirements of human beings: individuals' biological needs, coordinated social interaction, and group efficiency and survival. This assumption led Schwartz (1992) to form a model of 10 motivational value types. Table 1 lists the value types, their definitions, and their constituent values. As the table shows, each value type arises from the three universal human needs and can be expressed in several abstract values. For example, "benevolence" values are rooted in social interaction needs and express concern for the preservation and enhancement of the welfare of close others, and "self-direction" values are derived from organismic and interactional requirements and involve a preference for autonomy, creativity, and freedom.

More relevant to our aims, the domains can be arranged in a circular structure that reflects compatibilities and conflicts between values. In this structure, value domains that serve compatible motivational goals are adjacent, whereas value domains that serve incompatible motivational goals are opposite. For example, as shown in Fig. 1, values in the benevolence value domain (e.g., honest and loyal) are adjacent to values in the universalism domain (e.g., social justice and unity with nature), because both sets of values promote coordinated social interaction and group survival. Values in the benevolence value domain are plotted opposite to values in the achievement (e.g., successful and ambitious) domain, because achievement values promote personal welfare, rather than shared welfare with others. In contrast, values that are at right angles to benevolence values in this circular structure are held to serve conceptually orthogonal motives for innovation and progress (stimulation and self-direction values) or personal and group safety (security values). Consequently, people who consider benevolence values to be highly important should not be more or less likely to consider stimulation or tradition values to be highly important. This type of prediction from the circular model has received repeated support in smallest space analyses of ratings of the importance of values in over 200 samples from 60 countries (Sagiv \& Schwartz, 2000), supporting Schwartz's predictions about motivational conflicts and compatibilities between values. Because this model provides a single nomological net to merge diverse research on values across cultures, it could be argued that Schwartz's (1992) model of value structure is the most important recent development in research on values.

Table 1

Motivational Types of Values, Adapted from Schwartz (1992)

\begin{tabular}{|c|c|c|}
\hline Value domain & Definition & Motivational values \\
\hline Achievement & $\begin{array}{l}\text { Personal success through demonstrating competence according to social } \\
\text { standards }\end{array}$ & Successful, capable, intelligent, ambitious, influential \\
\hline Benevolence & $\begin{array}{l}\text { Preservation and enhancement of the welfare of people with whom one } \\
\text { is in frequent personal contact }\end{array}$ & $\begin{array}{l}\text { Helpful, honest, forgiving, true friendship, meaning in life, } \\
\text { loyal, mature love, responsible, spiritual life }\end{array}$ \\
\hline Conformity & $\begin{array}{l}\text { Restrain actions, inclinations, and impulses likely to upset or harm } \\
\text { others and violate social expectations or norms }\end{array}$ & Politeness, honouring parents, self-discipline, obedient \\
\hline Hedonism & Pleasure or sensuous gratification for oneself & Pleasure, enjoying life \\
\hline Power & $\begin{array}{l}\text { Social status and prestige, control or dominance over people and } \\
\text { resources }\end{array}$ & $\begin{array}{l}\text { Social power, authority, social recognition, wealth, } \\
\text { preserving my public image }\end{array}$ \\
\hline Security & Safety, harmony and stability of society, relationships, and the self & $\begin{array}{l}\text { Healthy, national security, social order, reciprocation of } \\
\text { favours, family security, clean, sense of belonging }\end{array}$ \\
\hline Self-Direction & Independent thought and action-choosing, creating, exploring & $\begin{array}{l}\text { Freedom, self-respect, independent, choosing own goals, } \\
\text { creativity, curious }\end{array}$ \\
\hline Stimulation & Excitement, novelty, and challenge in life & An exciting life, a varied life, daring \\
\hline Tradition & $\begin{array}{l}\text { Respect, commitment, and acceptance of the customs and ideas that } \\
\text { traditional culture or religion provide }\end{array}$ & $\begin{array}{l}\text { Devout, humble, moderate, detachment, respect for tradition, } \\
\text { accepting portion in life }\end{array}$ \\
\hline Universalism & $\begin{array}{l}\text { Understanding, appreciation, tolerance, and protection for the welfare } \\
\text { of all people and nature }\end{array}$ & $\begin{array}{l}\text { Social justice, wisdom, protecting the environment, a world } \\
\text { of beauty, equality, unity with nature, world at peace, broad- } \\
\text { minded, inner harmony }\end{array}$ \\
\hline
\end{tabular}




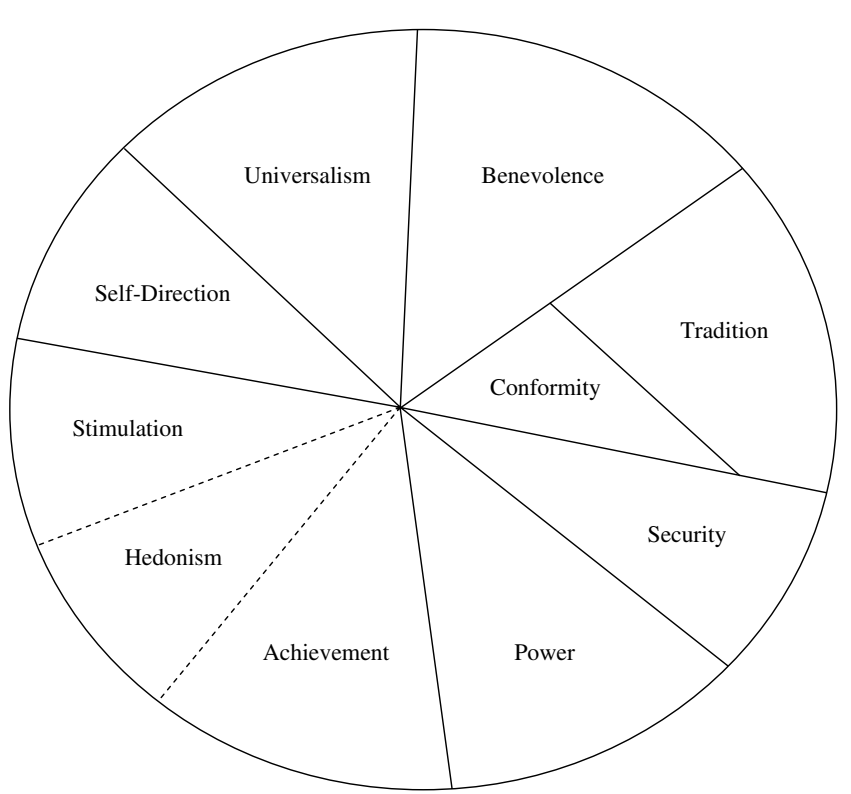

Fig. 1. The circular model of relations between motivational types of values (Schwartz, 1994, p.45). Dashed lines are used to delineate the region occupied by the value type "hedonism," because Schwartz (1992) indicates that this value type is related to both of the adjacent value types and can be considered as part of both.

\section{Unresolved Issues in Extant Tests of Inter-Value Structure}

Despite the abundant evidence supporting the circular model, there are important limitations on the extent to which the data actually reveal motivational conflicts and compatibilities between values. In other words, it is difficult to use the available evidence to extrapolate the real structure of values in people's minds. One obstacle is presented by the smallest space analyses themselves, which are limited in their utility for detecting circular representations (Fabrigar, Visser, \& Browne, 1997). Nonetheless, a more basic limitation is that the data rely exclusively on explicit value judgements. By themselves, explicit value judgements provide an important tool for identifying value importance, but their utility is attenuated by the fact that people may consciously adjust these ratings to be compatible with salient motives. For example, just as self-report measures of attitudes and personality can be affected by socially desirable response biases, so too can reports of values (Feather, 1975; Fisher \& Katz, 2000; Schwartz, Verkasalo, Antonovsky, \& Sagiv, 1997).

These value judgements can also be affected by the desire to appear consistent to the researcher and to the self. To maximize consistency, people may adjust their value ratings to be compatible with their theories about how each value conflicts with or supports the other values that they have just rated or are about to rate. People may be guided by their conscious theories of compatibility, similar to the way in which people use their implicit theories in autobiographical recall more generally (Ross \& McFarland, 1988). Value measurements may partly reflect conscious theories about conflicts and compatibilities between values, and these theories may or may not reflect the actual conflicts and compatibilities within the mental organization of values. This potential split between conscious theories of mental constructs and the actual organisation of them is now a virtual law of human social cognition (Gilbert, Pinel, Wilson, Blumberg, \& Wheatley, 2002; Nisbett \& Wilson, 1977) and memory (Carroll, Nelson, \& Kirwan, 1997; Leonesio \& Nelson, 1990) and provides a powerful argument for examining the mental organisation of values in a different way.

An additional argument for a novel approach is that the elicited patterns of relations between values may be driven more by access to shared semantic associations than by a parallel access to underlying goals. Quillian (1969) proposed that knowledge about the meaning of words and concepts is stored in a hierarchical network structure, wherein the meaning of a concept is defined partly by its relations to other concepts. That is, concepts are categorized in nodes in a hierarchical network and their meanings are defined not only by concepts stored in each node (e.g., canary and yellow), but also by concepts in a higher level of structure (e.g., bird). As a result, activation of a concept in memory spreads to connected nodes (Collins \& Loftus, 1975; Meyer, 1970), which enable people to judge more quickly the closeness of concepts when there are fewer nodes between them than when there are more nodes between them (Chang, 1986; Gold \& Robbins, 1979; Kounios, Montgomery, \& Smith, 1994). Also, retrieval of semantically related concepts is quicker than retrieval of concepts that are semantically unrelated (Collins \& Quillian, 1969; Thomsen, Lavine, \& Kounios, 1996).

The implications of this semantic network for the use of explicit value inter-relations are straightforward. People may begin a value survey by accessing (a) the meaning of the first value and (b) their motivation to achieve it. The activation of this value's meaning should then spread to values that have highly relevant semantic representations, including highly compatible and conflicting values. Ratings of these subsequent values could be adjusted up or down based on their semantic relations to the original value. That is, conceptual synonyms should be judged as being of similar importance, while conceptual antonyms should be judged as being of dissimilar importance. This process would not require access to the motivation or drive activated by each value; motivation could be accessed only during responses to the first few values (or even to the very first value alone). In other words, it could be argued that participants access their motivation regarding each value only at the outset of the survey and then use differences between their concept meanings to generate ratings of importance for subsequent values. So, the circular structure could represent differences between the meanings of values, rather than differences in the actual importance of values as guiding principles in the individuals' lives. The present research addresses these issues by examining the associations between values in memory. 


\section{Experiment 1}

Experiment 1 tested the circular structure of values by examining whether the speed of judging the importance of a value is affected by its motivational relation to a previously presented value, in a manner consistent with Schwartz's (1992) circular model. Abundant evidence indicates that the speed of judgement after a prior judgment reflects the degree of association between the two judgments in memory (Meyer \& Schvaneveldt, 1971; Ratcliff \& McKoon, 1981). Thus, if the circular model reflects the actual organisation of values in memory (and not just conscious theories), rating the importance of a value should facilitate faster ratings of a second value more strongly in pairs of values that the model holds to be highly compatible or conflicting than in pairs of values that are held to be unrelated. As assumed by the circular model, value distance was defined as the number of lower order value domains separating the two values in a pair (see Table 2).

\section{Method}

\section{Participants}

Forty-four undergraduate psychology students (30 women and $14 \mathrm{men}$ ) at Cardiff University participated for course credit.

\section{Procedure}

Participants took part individually. They were told that the session included two parts. First, they were asked to read a list of 48 values from the Schwartz (1992) Value Survey with the adjacent definitions of the values. After $5 \mathrm{~min}$ studying the text, participants were shown 24 trials on the computer screen, with 4 trials as practice. Each trial included two values, and each value appeared only once across the trials. Table 2 shows each value pair. The same pairs were presented across participants, but in different orders across trials. The order of presentation of values within the pairs was also counterbalanced across participants, such that participants received either "Domain 1" or "Domain 2" values first, as shown in Table 2.

Participants were asked to rate the importance of each value as a guiding principle in their lives, using keys labelled from 0 (not important to me) to 3 (extremely important to me). The presentation of the second value occurred immediately after rating the first value. To prevent interference across trials, participants were asked to count downward from a specific number (e.g., 87) in set decrements (e.g., 3) for $10 \mathrm{~s}$ after responding to each value pair. The starting numbers and decrements were different across the pairs, but the same for all participants. Sentences were presented using Medialab 2002, and reaction times were recorded using Direct Reaction Time Software 2002, which is able to record accurately to $1 \mathrm{~ms}$. After completing the experiment, participants were probed for suspicion and debriefed.

\section{Results and Discussion}

Multiple regression was used to examine the effect of the distance between value pairs on the time needed to rate the second value in the pair. Because our design involved many trials within-subjects, we could have employed within-subjects analyses and compared results across participants, between-subjects analyses and compared results across trials, or an analysis that utilized the variance within- and between-subjects (Michela, 1990). As recommended by Michela (1990), we chose to perform a curvilinear regression analysis that examined each trial across participants, while entering centred value distance, squared centred value distance (see Aiken \& West, 1991), and 43 dummy variables

Table 2

Value pairs that were used in the experiments

\begin{tabular}{|c|c|c|c|c|c|}
\hline Value 1 & Domain 1 & Value 2 & Domain 2 & Domain distance & Angle as distance \\
\hline Authority & Power & Wealth & Power & 0 & $7.5^{\circ}$ \\
\hline Creativity & Self-direction & Daring & Stimulation & 1 & $54.5^{\circ}$ \\
\hline Obedient & Conformity & Helpful & Benevolence & 2 & $41^{\circ}$ \\
\hline Having Fun & Hedonism & Clean & Security & 3 & $120.5^{\circ}$ \\
\hline Social-recognition & Power & Curious & Self-direction & 4 & $135.5^{\circ}$ \\
\hline Honest & Benevolence & Intelligent & Achievement & 5 & $170.5^{\circ}$ \\
\hline Family security & Security & Politeness & Conformity & 1 & $8^{\circ}$ \\
\hline An exciting life & Stimulation & Wisdom & Universalism & 2 & $52.5^{\circ}$ \\
\hline Healthy & Security & Happiness & Hedonism & 3 & $80^{\circ}$ \\
\hline Successful & Achievement & Moderate & Tradition & 4 & $111.5^{\circ}$ \\
\hline Broad-minded & Universalism & Social justice & Universalism & 0 & $45^{\circ}$ \\
\hline Detachment & Tradition & Honouring parents & Conformity & 1 & $10^{\circ}$ \\
\hline A Stimulating-Life & Stimulation & Capable & Achievement & 2 & $62^{\circ}$ \\
\hline Humble & Tradition & Independent & Self-direction & 3 & $156.5^{\circ}$ \\
\hline Equality & Universalism & National security & Security & 4 & $103^{\circ}$ \\
\hline Ambitious & Achievement & Loyal & Benevolence & 5 & $128.5^{\circ}$ \\
\hline Enjoying life & Hedonism & A varied life & Stimulation & 1 & $18.5^{\circ}$ \\
\hline Self-discipline & Conformity & Social power & Power & 2 & $114^{\circ}$ \\
\hline Freedom & Self-direction & Devout & Tradition & 3 & $135^{\circ}$ \\
\hline Forgiving & Benevolence & Pleasure & Hedonism & 4 & $174^{\circ}$ \\
\hline
\end{tabular}




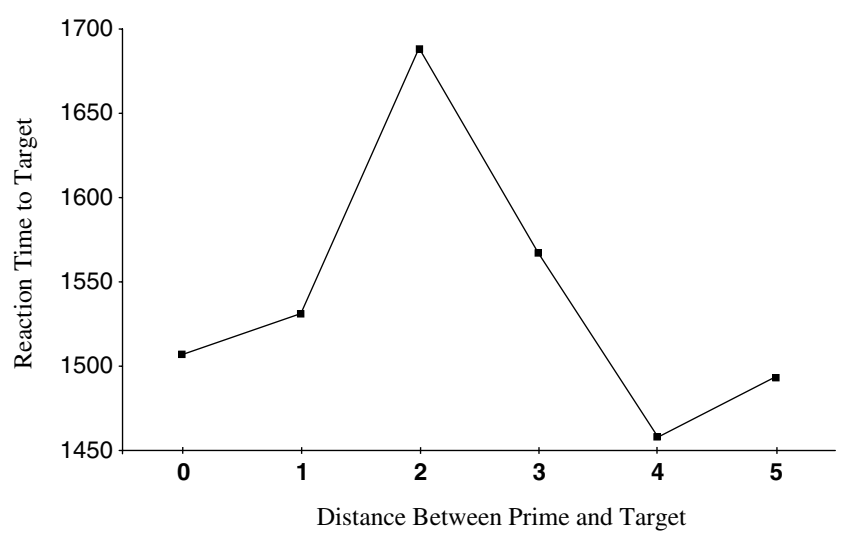

Fig. 2. Experiment 1: the curvilinear effect of value distance on the speed of value importance judgments.

(each representing one participant) as predictors of the time needed to rate the importance of the second value in each pair. The participant dummy variables helped to control for between-participants variability in the analysis, while utilizing all of the participants' response trials, and made it unnecessary to control for individual differences in response times to the values.

As hypothesised, the results revealed a significant negative curvilinear effect of value distance, $B=-48.41$, $t(753)=-2.49, p<.02$. The linear effect of value distance was not significant, $B=-12.11, t(754)=-.63$, $n s$. As shown in Fig. 2, participants took less time to rate the importance of the second value in pairs of values from the same and opposed domains than from unrelated domains. In other words, judgements of value importance were facilitated by prior judgement of a motivationally related value, regardless of whether its aim was similar to the target value or opposed to it. These findings support the circular model (Fig. 3).

\section{Experiment 2}

In Experiment 1, our examination of value structure utilized an identical prediction for values from adjacent and opposed value domains. An important exception should

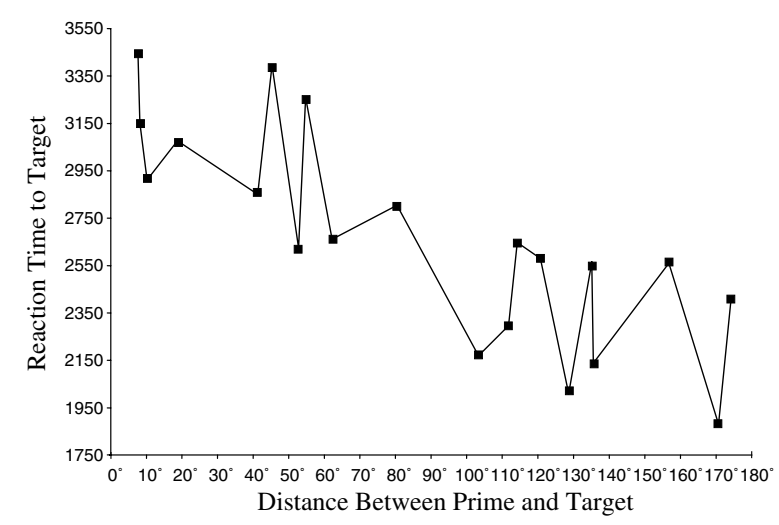

Fig. 3. Experiment 2: the linear effect of angle value distance on the speed of value preference judgments. occur when people are asked to choose which of two values is more important. In this relative judgment task, people should take longer to decide between two values that serve similar motives than to decide between two values that serve opposing motives. In both cases, it would be easy to access simultaneously the importance of each value in the pair, but the relative importance should be clearer when the values conflict. Values that serve compatible motives are often similar in importance, whereas values that serve opposing motives tend to be dissimilar in importance (Schwartz, 1992), making it easier to identify the more important value in the latter case. Again, as in Experiment 1, the key variable is not the actual importance of the value; it is the speed of the judgment. This time, however, a negative linear correlation between value distance and reaction time should occur. In other words, we predicted that participants would take more time to choose between the less distant values in a pair than between the more distant values in a pair, with reaction times for moderately distant value pairs in between.

A second aim of Experiment 2 was to use an additional, more direct measure of value distance. In Experiment 1, we defined value distance as the number of lower order value domains separating the two values in each pair. This operationalization relied on Schwartz's (1992) theoretical grouping of values into 10 lower order value domains. A more precise way of defining value distances is to measure the actual angle between the two values of each pair within the circular structure (see Table 2). We used the angle measure in Experiment 2. ${ }^{1}$

\section{Method}

\section{Participants}

Fifty-two students (33 female and 19 male) at Cardiff University participated for $£ 3$ payment.

\section{Procedure}

Participants were tested individually. They were told that the study includes two parts. First, they were asked to read a list of 48 values from the Schwartz (1992) Value Survey with the adjacent definitions of the values. After $5 \mathrm{~min}$ on this task, participants were shown 24 trials on the computer screen, with 4 trials as practice. Each trial presented one value on the left side of the screen and another one on the right side. The 20 pairs of values were constructed in the same manner as in Experiment 1 (see Table 2). Participants used two keys on the computer keyboard to indicate which value in each pair was more important to them as a guiding

\footnotetext{
${ }^{1}$ Unfortunately, we were not able to use the actual angles between the values of each pair as an additional distance measure in Experiment 1, because we did not record the sequence of presentation of the value pairs, which differed randomly between participants. Nonetheless, analyses of actual angle distances in Experiments 2 and 3 revealed the same effects as in Experiment 1. Thus, for the sake of brevity, our description of Experiments 2 and 3 focuses on the more precise angle measure.
} 
principle in their lives, and their time to make each choice was recorded using Direct Reaction Time Software 2002. After completing this task, participants were probed for suspicion and debriefed.

\section{Results and Discussion}

Similar to Experiment 1, we conducted a curvilinear regression analysis that utilized centred value distance, squared centred value distance, and 51 dummy variables (each representing one participant) as predictors of reaction times to the value choices. Results indicated a significant negative linear effect of value distance, $B=-350.00$, $t(987)=-9.01, p<.001$, and the curvilinear effect of value distance was not significant, $B=45.41, t(986)=.98, n s$. That is, participants took more time to react to trials including highly related values than to trials including opposing values, with reaction times to unrelated values falling in between. These results are consistent with the circular model, because it should take more time to choose a value from a pair of values that serve the same motive than between values that serve opposing, distinct goals of different importance to the individual.

\section{Experiment 3}

Thus far, the results of these experiments are consistent with Schwartz's (1992) predictions about motivational compatibility and conflict between values. Nonetheless, the results do not rule out an effect of shared semantic representations. As indicated in the introduction, we expect that the circular model is a joint function of latent motivational compatibility and conflict and of the semantic associations between values. To provide clearer support for the role of motivational relations, it is important to show that predicted value compatibilities and conflicts predict the speed of value judgement independently of participants' perceptions of semantic relatedness. This issue was investigated in our third experiment. Experiment 3 addressed this aim using a paradigm that was a modification of our first experiment, by including our measure of angle distances separating values and a new measure, which asked participants to indicate the semantic relatedness of pairs of values. In Experiment 3, participants completed the value importance trials from Experiment 1 and then judged the semantic relatedness of the same value pairs. We expected to find a curvilinear effect of value distance on speed of value judgement, as in Experiment 1. Moreover, we expected that this effect would remain significant after controlling for participants' judgements of semantic relatedness.

\section{Method}

\section{Participants}

Forty-eight undergraduate psychology students (31 female and 17 male) at Cardiff University participated for course credit.

\section{Procedure}

Participants took part individually. They were told that the study included three parts. First, they were asked to read a list of 46 values from the Schwartz (1992) Value Survey, with the adjacent definitions of the values. ${ }^{2}$ After $5 \mathrm{~min}$ studying the text, participants completed 23 value importance trials from Experiment 1. As in Experiment 1, reaction times for the second value in each pair were recorded for our principal analysis. In a third task, participants completed judgements of the values' semantic relatedness. That is, for each value pair, participants were asked to rate the extent to which the values were related in meaning, using a 4-point scale on the computer keyboard from 0 (not related) to 3 (extremely related). The speed of this rating was recorded. After completing this task, participants were probed for suspicion and debriefed. The instruments for presenting the trials and recording data were the same as in Experiment 1, although we also took steps to use the actual angles between the two values of each value pair as a more precise measure of value distance (see Footnote 1).

\section{Result and Discussion}

We first conducted a regression analysis with centred value distance, squared centred value distance, and 47 dummy variables (each representing one participant) as predictors of the speed of rating the second value in each pair. As expected, the results indicated a significant negative curvilinear effect of value distance, $B=-103.49$, $t(766)=-4.49, p<.001$, and the linear effect of value distance was not significant, $B=18.34, t(767)=1.02, n s$.

We then examined the effect of perceived semantic relatedness on the speed of rating the second value in each pair. In this analysis, we entered centred perceived semantic relatedness, squared centred perceived semantic relatedness, and 47 dummy variables (each representing one participant) as predictors of the speed of rating of the second value in each pair. Consistent with the broader literature on semantic memory effects, the results revealed a significant negative linear relation between perceived semantic relatedness and the speed of rating the second values, $B=-97.64$, $t(810)=-5.58, p<.001$, strongly supporting the validity of our measure. We did not expect a curvilinear effect of semantic relatedness on reaction times, and this effect was not obtained, $B=-11.90, t(809)=-.61, n s$.

To further examine the validity of the semantic relatedness measure, we correlated perceived semantic relatedness with the discrepancy in value importance between the two values of each pair. Based on the assumption that semantically related values should be perceived as similarly important, we hypothesized a negative relation between semantic relatedness and value discrepancies, and this prediction was supported by our data, $r(862)=-.26, p<.001$. Thus, this

\footnotetext{
2 The deleted values for Experiment 3 were "detachment" and "honouring parents".
} 
evidence provided a second source of support for the validity of our measure of semantic relatedness. ${ }^{3}$

Our main hypothesis was that the effect of value distance on the speed of judgement of the second values would be at least partly independent of the effect of perceived semantic relatedness. A regression analysis tested this prediction by entering value distance, squared value distance, perceived semantic relatedness, squared perceived semantic relatedness (all measures centred), and 47 dummy variables (each representing one participant) as predictors of the speed of rating of the second value in each pair. The results indicated no significant linear effect of value distance, $B=-28.82$, $t(811)=-1.48, n s$, but a significant and independent linear effect of perceived semantic relatedness, $B=-112.12$, $t(811)=-5.75, p<.001$. More important, there was an independent curvilinear effect of value distance, $B=-94.90$, $t(811)=-4.12, p<.001$, and the curvilinear effect of semantic relatedness was not significant, $B=-20.59$, $t(807)=-1.03$, ns. Thus, semantic relatedness failed to account for the curvilinear effect of value distance on the speed of value judgements. ${ }^{4}$

\section{General Discussion}

The purpose of the present experiments was to investigate the latent structure of social values in memory. Experiments 1 and 2 obtained results consistent with Schwartz's (1992) circular model of motivational relations between values. That is, the experiments found that the speed of value ratings (Experiment 1) and value preference judgments (Experiment 2) in pairs of values could be predicted from the compatibilities and conflicts between values in the circular model. Experiment 3 found that the hypothetical compatibilities and conflicts predicted the speed of value importance judgments over and above the effect of the perceived semantic relations among values.

As we noted earlier, this evidence is important partly because the circular model has the potential to help integrate a large amount of evidence about antecedents and consequences of values. Past support for the circular model relied mostly on theoretically consistent correlations between self-reports of value importance, and these correlations could have been driven by participants' conscious the-

\footnotetext{
${ }^{3}$ Additionally, we found that speed of judgement of the second value was facilitated by smaller discrepancies in value importance between the two values of each value pair, $r(862)=.12, p<.001$. The same was also true for Experiment 1, $r(797)=.10, p<.001$.

${ }^{4}$ Though not directly germane to our assessment of inter-value structure in memory, we tested whether values that are more closely located in the circular structure are rated as less discrepant in importance. As expected, there was a significant positive correlation between value distance and the absolute magnitude of discrepancies in value importance across value pairs, $r(814)=.07, p<.05$. This result also occurred in Experiment 1, $r(818)=.14, p<.001$, using the simple count of lower order value domains that separated values in each pair. These results are consistent with the prior examinations of value structure, which used value distance and self-reports of value importance as variables.
}

ories about value conflicts and by their perceptions of semantic relations between values. The present data do not possess these limitations and provide a novel source of support for the circular model. At the same time, we obtained evidence regarding two different sources of the obtained value relations: motivational relatedness and semantic relatedness. The results provide stronger evidence for a dynamic conceptual structure of values based on their relation to motives that vary in compatibility.

Taken together, the results of our experiments strongly support the validity of Schwartz's (1992) circular model. However, this evidence does not indicate that the circular model is without limitations. For example, Schwartz (1992) reports that 44 of the 56 values in the circular model show a consistent location in a specified lower order value domain across cultures. ${ }^{5}$ The lack of consistency for 12 of the values suggests that it is important to take cultural and group differences into consideration when it comes to relations between values and value dependent constructs (e.g., attitudes and behaviour).

Nevertheless, the present results have potential implications for understanding value-related behaviour. Of particular relevance is our discovery of close ties in memory between motivationally compatible values. Given this result, it is likely that priming a single value should often activate other related values. As a consequence, value activation may have complex effects on value-related attitudes and behaviours that have not yet been detected. For example, priming "national security" may affect behaviours that are related to similar conservation motives (e.g., devotion to a custom or tradition), but are not directly related to the value itself. This potential impact can be predicted only by understanding the associations between values in memory.

It is worth noting that such effects could not be detected in past research, because past research has focused on activating one value and measuring one behaviour. For example, in past research examining "automaticity" processes in behaviour, experiments have shown that subliminally activation of different values can increase the performance of relevant behaviours that support the value (Bargh, Gollwitzer, Lee-Chai, Barndollar, \& Trotschel, 2001; Macrae \& Johnston, 1998). Similar effects of priming single values have been obtained in other research focusing on the values concept (Roccas, 2003; Verplanken \& Holland, 2002). In contrast, our experiments examined a variety of values for each participant and found that priming a value increases the activation of closely related values. Nonetheless, it remains to be seen whether value priming operates in the same manner when other values are not simultaneously salient, and it is worth considering that inter-value associations may vary between cultures and between participants in a culture. These issues merit further research.

\footnotetext{
${ }^{5}$ Of the 38 values used across our experiments (see Table 2), seven were not included in Schwartz's (1992) list of the 44 values that have highly stable inter-value relations across cultures: having fun, social recognition, intelligent, healthy, happiness, detachment, and a stimulating life.
} 
To summarise, the present experiments revealed strong associations between values in memory that are consistent with the motivational compatibilities and conflicts posited in the circular model of values, while showing that perceived semantic relations are not sufficient to explain the associations between values in memory. These results indicate that it may be problematic to focus on single values in isolation. Through their existence within a larger motivational network, the impact of social values on behaviour may be intricate and far-reaching. The present research strengthens the empirical foundation for predicting these diverse effects.

\section{References}

Aiken, L. S., \& West, S. G. (1991). Multiple regression: Testing and interpreting interactions. Newbury Park, CA: Sage.

Bardi, A., \& Schwartz, S. H. (2003). Values and behavior: Strength and structure of relations. Personality and Social Psychology Bulletin, 29, $1207-1220$.

Bargh, J. A., Gollwitzer, P. M., Lee-Chai, A., Barndollar, K., \& Trotschel, R. (2001). The automated will: Nonconscious activation and pursuit of behavioural goals. Journal of Personality and Social Psychology, 81, 1014-1027.

Carroll, M., Nelson, T. O., \& Kirwan, A. (1997). Trade off of semantic relatedness and degree of over learning: Differential effects on metamemory and on long-term retention. Acta Psychologica, 95, 239-253.

Chang, T. M. (1986). Semantic memory: Facts and models. Psychological Bulletin, 99, 199-220.

Collins, A. M., \& Loftus, E. D. (1975). A spreading activation theory of semantic memory. Psychological Review, 82, 407-428.

Collins, A. M., \& Quillian, M. R. (1969). Retrieval time from semantic memory. Journal of Verbal Learning and Verbal Behaviour, 8, 240-247.

Fabrigar, L. R., Visser, P. S., \& Browne, M. W. (1997). Conceptual and methodological issues in testing the circumplex structure of data in personality and social psychology. Personality and Social Psychology Review, 1, 184-203.

Feather, N. T. (1975). Values in education and society. New York: Free Press.

Feather, N. T. (1992). Values, valences, expectations, and actions. Journal of Social Issues, 48, 109-124.

Fisher, R. J., \& Katz, J. E. (2000). Social-desirability bias and the validity of self-reported values. Psychology and Marketing, 17, 105-120.

Gilbert, D. T., Pinel, E. C., Wilson, D. T., Blumberg, S. J., \& Wheatley, T. (2002). Durability bias in affective forecasting. In D. Griffin \& T. Gilovich (Eds.), Heuristics and biases: The psychology of intuitive judgement (pp. 292-312). New York, NY: Cambridge University Press.

Gold, J. A., \& Robbins, M. A. (1979). Attitudes and values: A further test of the semantic memory model. Journal of Social Psychology, 108, 75-81.

Kounios, J., Montgomery, E. C., \& Smith, R. W. (1994). Semantic memory and the granularity of semantic relations: Evidence from speed-accuracy decomposition. Memory and Cognition, 22, 729-741.

Leonesio, R. J., \& Nelson, T. O. (1990). Do different metamemory judgements tap the same underlying aspects of memory? Journal of Experimental Psychology: Learning, Memory and Cognition, 16, 464 470.

Macrae, C., \& Johnston, L. (1998). Help, I need somebody: Automatic action and inaction. Social Cognition, 16, 400-417.

Maio, G. R., \& Olson, J. M. (1995). Relations between values, attitudes, and behavioral intentions: The moderating role of attitude function. Journal of Experimental Social Psychology, 31, 266-285.
Maio, G. R., \& Olson, J. M. (1998). Values as truisms: Evidence and implications. Journal of Personality and Social Psychology, 74, 294-311.

Meyer, D. E. (1970). On the representation and retrieval of stored semantic information. Cognitive Psychology, 1, 242-300.

Meyer, D. E., \& Schvaneveldt, R. W. (1971). Facilitation in recognizing pairs of words: Evidence of a dependence between retrieval operations. Journal of Experimental Psychology: General, 90, 227-234.

Michela, J. L. (1990). Within-person correlational design and analysis. In C. Hendick \& M. S. Clark (Eds.), Research methods in personality and social psychology (Vol. 11, pp. 279-311). Thousand Oaks, CA: Sage Publications, Inc.

Nisbett, R. E., \& Wilson, T. D. (1977). Telling more than we can know: Verbal reports on mental process. Psychological Review, 84, 231-259.

Olson, J. M., \& Maio, G. R. (2003). Attitudes in social behaviour. In M. J. Lerner \& T. Millon (Eds.), Handbook of psychology: Personality and social psychology. New York, NY: John Wiley and Sons, Inc.

Quillian, M. R. (1969). The teachable language comprehender: A simulation program and theory of language. Communication of the ACM, 12, 459-476.

Ratcliff, R., \& McKoon, G. (1981). Does activation really spread? Psychological Review, 88, 454-462.

Roccas, S. (2003). Identification and status revisited: The moderating role of self-enhancement and self-transcendence values. Personality and Social Psychology Bulletin, 29, 726-736.

Rohan, M. J. (2000). A rose by any name? The values construct. Personality and Social Psychology Review, 4, 255-277.

Rokeach, M. (1973). The nature of human values. New York, NY: Free Press.

Rokeach, M. (1985). Inducing change and stability in belief systems and personality structures. Journal of Social Issues, 41, 153-171.

Rokeach, M., \& Ball-Rokeach, S. J. (1989). Stability and change in American value priorities. American Psychologist, 44, 775-784.

Ross, M., \& McFarland, C. (1988). Constructing the past: Biases in personal memories. In A. W. Kruglanski \& D. Bar-Tal (Eds.), The social psychology of knowledge (pp. 299-314). New York, NY: Cambridge University Press.

Sagiv, L., \& Schwartz, S. H. (2000). Value priorities and subjective wellbeing: Direct relations and congruity effects. European Journal of Social Psychology, 30, 177-198.

Schonpflug, U. (2001). Intergenerational transmission of values. Journal of Cross-Cultural Psychology, 32, 174-185.

Schwartz, S. H. (1992). Universals in the content and structure of values: Theoretical advances and empirical tests in 20 countries. In M. Zanna (Ed.), Advances in Experimental Social Psychology (Vol. 25, pp. 1-65). San Diego, CA: Academic Press.

Schwartz, S. H. (1994). Are there universal aspects in the structure and contents of human values? Journal of Social Issues, 50, 19-45.

Schwartz, S. H., Verkasalo, M., Antonovsky, A., \& Sagiv, L. (1997). Value priorities and social desirability: Much substance, some style. British Journal of Social Psychology, 36, 3-18.

Thomsen, C. J., Lavine, H., \& Kounios, J. (1996). Social value and attitude concepts in semantic memory: Relational structure, concept strength, and the fan effect. Social Cognition, 14, 191-225.

Verplanken, B., \& Holland, R. W. (2002). Motivated decision making: Effects of activation and self-centrality of values on choices and behavior. Journal of Personality and Social Psychology, 82, 434-447.

Whitbeck, L. B., \& Gecas, V. (1988). Value attributions and value transmission between parent and child. Journal of Marriage and the Family, 50, 829-840.

Yik, M. S. M., \& Tang, C. S. (1996). Linking personality and values. Personality and Individual Differences, 21,767-774. 